\title{
PREPAYMENT OPTION OF A PERPETUAL CORPORATE LOAN: THE IMPACT OF THE FUNDING COSTS
}

\author{
TIMOTHEE PAPIN* AND GABRIEL TURINICI ${ }^{\dagger}$
}

\begin{abstract}
We investigate in this paper a perpetual prepayment option related to a corporate loan. The short interest rate and default intensity of the firm are supposed to follow CIR processes. A liquidity term that represents the funding costs of the bank is introduced and modeled as a continuous time discrete state Markov chain. The prepayment option needs specific attention as the payoff itself is a derivative product and thus an implicit function of the parameters of the problem and of the dynamics. We prove verification results that allows to certify the geometry of the exercise region and compute the price of the option. We show moreover that the price is the solution of a constrained minimization problem and propose a numerical algorithm building on this result. The algorithm is implemented in a two-dimensional code and several examples are considered. It is found that the impact of the prepayment option on the loan value is not to be neglected and should be used to assess the risks related to client prepayment. Moreover the Markov chain liquidity model is seen to describe more accurately clients' prepayment behavior than a model with constant liquidity.
\end{abstract}

Key words. funding costs, liquidity regime, loan prepayment, mortgage option, American option, perpetual option, option pricing, variational inequality, prepayment option, CIR process, switching regimes, Markov modulated dynamics.

AMS subject classifications. 91G20, 91G30, 91G40, 91G50, 91G60, 91G80, 93E20

1. Introduction. A loan contract issued by a bank for its corporate clients is a financial agreement that often includes a prepayment option which entitles the client, if he desires so, to pay a fraction (or up to 100\%) of its loan earlier than the maturity. The company prepays when its credit profile improves so that it can refinance its debt at a cheaper rate.

In order to decide whether the exercise of the option is worthwhile the company compares the actualized value of the remaining payments with the nominal value of the loan, denoted by $K$. If the former exceeds the latter nominal then it is optimal to prepay.

When the interest rates are not constant or the borrower is subject to default the computation of the actualization is less straightforward. It starts with considering all possible scenarios of evolution for interest rate and default intensity in a riskneutral framework in order to compute the average value of remaining payments (including the final payment of the principal if applicable); this quantity will be called "PVRP"(denoted $\xi$ ) and is the present value of the remaining payments i.e., the cash amount equivalent of the value of remaining payments. Then $\xi$ is compared with the nominal $K:$ if $\xi \geq K$ then the borrower should prepay, otherwise not. Recall that at the initial time the payments correspond to a rate, the sum of the (variable) short term interest rate (e.g., LIBOR or EURIBOR) and a contractual margin $\overline{\rho_{0}}$ chosen such that $\xi=K$ at origination. Note that in order to compute the price of the embedded prepayment option the lender also uses the PVRP as it will be seen below.

The bank that proposes the loan finances it through a bond program (possibly mutualized for several loans) at some spread depending on its own credit profile and market conditions. In order for the corporate loan to be profitable the rate of the bond, that is also indexed on LIBOR or EURIBOR, has to be lower than the rate of

* BNP Paribas CIB Ressource Portfolio Management and CEREMADE, Université Paris Dauphine

${ }^{\dagger}$ CEREMADE, Université Paris Dauphine (Gabriel.Turinici@dauphine.fr) 
the loan. This condition is easy to check at the origination of both contracts and is always enforced by the bank. However if the client prepays the bank finds itself in a non-symmetric situation: the periodic interest payments from client is terminated but the bank still has to pay the interests and principal of its own bond; the bond does not have a prepayment option or such an option is costly. The risk is that the amount $K$ received from the client at prepayment time cannot be invested in another product with interest rate superior to that of the bond.

Thus a first question is how should the bank funds its corporate loans and what provisions are to be made to handle the prepayment risk. This is a valuation problem.

An even more important question whether it is possible that many clients decide to prepay at the same time. This circumstance can happen for instance when a crisis is over and clients can again borrow at 'normal', lower, rates. We address this question by introducing liquidity regimes to model funding costs.

Liquidity is crucial to the stability of the financial system and can cause bank failures if systemic liquidity squeezes appear. Historical events like the Asian crisis of 1997; the Russian financial crisis of 1998; the defaults of hedge funds and investment firms like LTCM, Enron, Worldcom and Lehman Brothers; sovereign debts crisis of 2010-11, prove that banks hold significant liquidity risk in their balance sheets. Even if liquidity problems have a very low probability to occur, a liquidity crisis can have a severe impact on a bank's funding costs, its market access (reputation risk) and short-term funding capabilities.

Following the state of the economic environment, the liquidity can be defined by distinct states. Between two crises, investors are confident and banks find it easy to launch their long term refinancing programs through regular bonds issuances. Thus the liquidity market is stable. On the contrary, during crisis liquidity becomes scarce, pushing the liquidity curve to very high levels; the transition between these two distinct regimes is often sudden.

In order to model the presence of distinct liquidity regimes we will simulate the liquidity cost by a continuous time observable Markov chain that can have a discrete set of possible values, one for each regime. It is seen (cf. Section 4.5) that considering several liquidity regimes explains better clients' prepayment behavior than a constant liquidity model.

In practice it is interesting to study long-term loans that are set for more than three years and can run for more than twenty years. Note that the longest the maturity of the loan, the riskier the prepayment option. The perpetual options (i.e., with infinite time to maturity) are the object of this paper and provide a conservative estimation of the prepayment risk of any loan.

From a technical point of view this paper faces several non-standard conditions: although the goal is to value a perpetual American option the payoff of the option is highly non-standard (is dependent on the $P V R P$ which is itself a derivative product). As a consequence the characterization of the exercise region is not standard and technical conditions have to be met. Furthermore our focus here is on a specific type of dynamics (of CIR type) with even more specific interest on the situation when several funding regimes are present.

The balance of the paper is as follows: in the remainder of this section (SubSection 1.1) we review the related existing literature; in Section 2 we prove a first theoretical result that allows to identify the exercise region. In Section 3 we show that the price is the solution to some constraint optimization problem which allows to construct a numerical algorithm. A 2D numerical implementation of the algorithm 
is the object of the Section 4 and several examples are presented.

1.1. Related literature. There exist few articles (e.g., works by Cossin et al. [10]) on the loan prepayment option but a close subject, the prepayment option in a fixed-rate mortgage loan, has been covered in several papers by Hilliard and Kau [17] and more recent works by Chen et al. [8] that express the prepayment option as a function depending on two state variables : interest rate and house price. Their approach is based on a bivariate binomial option pricing technique with a stochastic interest rate and a stochastic house value (CIR processes). Although the trinomial tree may also be computationally interesting and relevant for this problem so far no numerical implementations were proposed, to the best of our knowledge, for this specific problem.

Another contribution by Cossin et al. [10] applies the binomial tree technique (but of course it is time-consuming for long-term loans due to the nature of binomial trees) to corporate loans. They consider a prepayment option associated to a 1 year loan with a quarterly step but it is computationally difficult to have an accurate assessment of the option price for a 10 years loan.

There also exist mortgage prepayment decision models based on Poisson regression approach for mortgage loans. See, for example, Schwartz and Torous [29]. Unfortunately, the volume and history of data are very weak in the corporate loan market to obtain reliable results.

Due to the structure of their approach none of these papers investigated rigorously the geometry of the exercise region because it was explicitly given by some numerical algorithm (which was supposed to converge). This cannot be avoided any more in our case and requires that particular care be taken when stating the optimality of the solution. Furthermore, to the best of our knowledge, none of these approaches explored the prepayment option with several regimes present.

The analysis of Markov-modulated regimes has been investigated in the literature when the underlying(s) follow the Black\& Scholes dynamics with drift and volatility having Markov jumps; several works are of interest in this area: Guo and Zhang [34] have derived the closed-form solutions for vanilla American put; Guo analyses in [15] Russian (i.e., perpetual look-back) options and is able to derive explicit solutions for the optimal stopping time; in [32] $\mathrm{Xu}$ and $\mathrm{Wu}$ analyse the situation of a two-asset perpetual American option where the pay-off function is a homogeneous function of degree one; Mamon and Rodrigo [25] find explicit solutions to vanilla European options. Buffington and Elliott [5] study European and American options and obtain equations for the price. A distinct approach (Hopf factorization) is used by Jobert and Rogers [20] to derive very good approximations of the option prices for, among others, American puts. Other contributions include [33, 31].

A different class of contributions discuss the liquidity; among them several contributions point out that the liquidity displays "regimes" i.e. a finite list of distinctive macro-economic circumstances, see for instance $[12,24]$ and references within. Our situation corresponds precisely to this view as it will be seen in Section 2 .

Works involving Markov switched regimes and CIR dynamics appears in [14] where the bond valuation problem is considered (but not in the form of an American option; their approach will be relevant to the computation of the payoff of our American option although in their model only the mean reverting level is subject to Markov jumps) and in [35] where the term structure of the interest rates is analyzed. A relevant connected work is [30] where the bond price is obtained when the short rate process is governed by a Markovian regime-switching jump-diffusion version of 
the Vasicek model; the authors provide in addition the suitable mathematical arguments to study piecewise Vasicek dynamics (here the dynamics is still piecewise but CIR).

On the other hand numerical methods are proposed in [18] where it is found that a fixed point policy iteration coupled with a direct control formulation seems to perform best.

The pricing of a simplified one-dimensional model with constant interest rate was proposed in [27]. With respect to this first work the present contribution does not only investigate the non-trivial dynamics of the interest rate but also proposes an adequate numerical algorithm (in Section 3 ) that requires at its turn the introduction of adapted functional spaces.

Finally, we refer to [19] for theoretical results concerning the pricing of American options in general.

This work contributes in several ways to the existing literature:

- first by exploring the prepayment option when several regimes are present

- secondly by analyzing a Markov-modulated CIR dynamics with a non-standard payoff

- thirdly by proposing a verification theorem revealing a technical assumption not present in the literature

- finally, by introducing a rigorous framework for a numerical algorithm to compute the option price. In particular we identify the relevant weighted Sobolev functional space that allows to formulate a well-posed associated variational inequality.

\section{Perpetual prepayment option: the geometry of the exercise region.}

2.1. The risk neutral dynamics. The prepayment option depends on three distinct dynamics:

- the (short) interest rate $r_{t}$, which follows a piecewise CIR (Cox-IngersollRoss) process (see [6, 1, 22, 23, 3] for theoretical and numerical aspects of CIR processes and the situations where the CIR process has been used in finance and especially to model the short interest rates);

- the default intensity $\lambda_{t}$ which also follows a piecewise CIR process; the CIR process has already been used to model the default intensity, we refer to [23, 3] for details.

- the liquidity $l_{t}$ which depends on the economic environment and jumps among a finite list of states; it is described by a finite state Markov chain $\mathbb{X}=$ $\left\{X_{t}, t \geq 0\right\}$. See $[12,24]$ for modeling issues. The state space $\mathbb{X}$ can be taken to be, without loss of generality, the set of unit vectors $E=\left\{e_{1}, e_{2}, \ldots, e_{N}\right\}$, $e_{i}=(0, \ldots, 0,1,0, \ldots, 0)^{T} \in \mathbb{R}^{N}$. Here $T$ is the transposition operator.

As the purpose of this paper is not to introduce a new model but rather to put on firm ground the valuation of the prepayment option we refer to the cited works for an explanation of why this risk-neutral model is suitable to this circumstance.

Assuming the process $X_{t}$ is homogeneous in time and has a rate matrix $A$, then

$$
X_{t}=X_{0}+\int_{0}^{t} A X_{u} d u+M_{t}, X_{0}=\overline{X_{0}},
$$

where $\mathbf{M}=\left\{M_{t}, t \geq 0\right\}$ is a martingale with respect to the filtration generated by $\mathbb{X}$. In differential form

$$
d X_{t}=A X_{t} d t+d M_{t}, X_{0}=\overline{X_{0}} .
$$


We assume the instantaneous liquidity cost of the bank $l_{t}$ is positive and depends on the state $X_{t}$ of the economy, so that

$$
l_{t}=\left\langle 1, X_{t}\right\rangle
$$

for some constant vector $\mathbf{l}$ that collects the numerical values of liquidity for all regimes $e_{k} \in E$.

Denote by $a_{k, j}$ the entry on the line $k$ and the column $j$ of the $N \times N$ matrix $A$ with $a_{k, j} \geq 0$ for $j \neq k$ and $\sum_{j=1}^{N} a_{k, j}=0$ for any $k$.

We model the intensity dynamics by a CIR process with parameters depending on the regime $X_{t}$ :

$$
\begin{aligned}
& d \lambda_{t}=\gamma_{\lambda}\left(X_{t}\right)\left(\theta_{\lambda}\left(X_{t}\right)-\lambda_{t}\right) d t+\sigma_{\lambda}\left(X_{t}\right) \sqrt{\lambda_{t}} d W_{t}, \lambda_{0}=\overline{\lambda_{0}}, \\
& \gamma_{\lambda}\left(X_{t}\right), \theta_{\lambda}\left(X_{t}\right), \sigma_{\lambda}\left(X_{t}\right)>0 .
\end{aligned}
$$

The short rate $r$ also follows a CIR process with parameters depending on the regime $X_{t}$ :

$$
\begin{aligned}
& d r_{t}=\gamma_{r}\left(X_{t}\right)\left(\theta_{r}\left(X_{t}\right)-r_{t}\right) d t+\sigma_{r}\left(X_{t}\right) \sqrt{r_{t}} d Z_{t}, r_{0}=\overline{r_{0}}, \\
& \gamma_{r}\left(X_{t}\right), \theta_{r}\left(X_{t}\right), \sigma_{r}\left(X_{t}\right)>0 .
\end{aligned}
$$

In order to ease the notations we may sometimes write $\gamma_{\lambda, k}$ instead of $\gamma_{\lambda}\left(e_{k}\right)$ and similar notations for $\sigma_{\lambda}\left(e_{k}\right), \theta_{\lambda}\left(e_{k}\right), \gamma_{r}\left(e_{k}\right), \sigma_{r}\left(e_{k}\right)$ and $\theta_{r}\left(e_{k}\right)$ for $k=1, \ldots, N$.

It is known that if

$$
2 \gamma_{\lambda, k} \theta_{\lambda, k} \geq \sigma_{\lambda, k}^{2}, \quad \forall k=1, \ldots, N
$$

then the intensity $\lambda_{t}$ is strictly positive at all times. We assume that the condition (2.8) is satisfied. Same hypothesis is assumed for the short rate dynamics:

$$
2 \gamma_{r, k} \theta_{r, k} \geq \sigma_{r, k}^{2}, \quad \forall k=1, \ldots, N .
$$

Here $W_{t}$ and $Z_{t}$ are two Brownian motions independent of the filtration generated by $\mathbb{X}$. Their correlation is possibly non-null but constant i.e., with usual notations

$$
\left\langle W_{t}, Z_{t}\right\rangle=\rho t,|\rho| \leq 1 .
$$

We obtain thus the following joint dynamics which is supposed to be the relevant risk-neutral dynamics for the valuation of the prepayment option:

$$
d\left(\begin{array}{c}
X_{t} \\
\lambda_{t} \\
r_{t}
\end{array}\right)=\left(\begin{array}{c}
A X_{t} \\
\gamma_{\lambda}\left(X_{t}\right)\left(\theta_{\lambda}\left(X_{t}\right)-\lambda_{t}\right) \\
\gamma_{r}\left(X_{t}\right)\left(\theta_{r}\left(X_{t}\right)-r_{t}\right)
\end{array}\right) d t+\left(\begin{array}{c}
d M_{t} \\
\sigma_{\lambda}\left(X_{t}\right) \sqrt{\lambda_{t}} d W_{t} \\
\sigma_{r}\left(X_{t}\right) \sqrt{r_{t}} d Z_{t}
\end{array}\right),\left(\begin{array}{c}
X_{0} \\
\lambda_{0} \\
r_{0}
\end{array}\right)=\left(\begin{array}{c}
\overline{X_{0}} \\
\overline{\lambda_{0}} \\
\overline{r_{0}}
\end{array}\right) .
$$

REMARK 1. The selection of a risk-neutral dynamics (or equivalently of a pricing measure) is not a trivial task in general and even less for incomplete markets (see [7] for further details). 
2.2. The PVRP. Consider a loan with an initial contractual margin $\overline{\rho_{0}}$ calculated at the origination to match the par value $K$ of the loan. At time $t$ the client firm pays interests at rate $r_{t}+\overline{\rho_{0}}$. Let $\xi\left(t, T, r_{t}, \lambda_{t}, X_{t}\right)$ be the present value of the remaining payments at time $t$ of the corporate loan with contractual maturity $T$ (the interested reader can consult $[23,3]$ and references within for additional information related to $\xi)$.

A quantity that is meaningful for the bank is the "loan value" $L V(t, T, r, \lambda, X)$ defined as $\xi(t, T, r, \lambda, X)$ minus the prepayment option value $P(t, T, r, \lambda, X)$.

$$
L V(t, T, r, \lambda, X)=\xi(t, T, r, \lambda, X)-P(t, T, r, \lambda, X) .
$$

The PVRP $\xi$ is the present value of the cash flows discounted at the instantaneous risky rate, where the instantaneous risky rate at time $t$ is the short rate $r_{t}$ plus the liquidity cost $l_{t}$ plus the intensity $\lambda_{t}$. We obtain for $r>0, \lambda>0$ that the $P V R P$ is :

$$
K \mathbb{E}\left[\int_{t}^{T}\left(r_{s}+\overline{\rho_{0}}\right) e^{-\int_{t}^{s}\left(r_{u}+l_{u}+\lambda_{u}\right) d u} d s+e^{-\int_{t}^{T}\left(r_{s}+l_{s}+\lambda_{s}\right) d s} \mid r_{t}=r, \lambda_{t}=\lambda, X_{t}=X\right] .
$$

For a perpetual loan $T=+\infty$ and since $r_{t}>0, \lambda_{t}>0$ we obtain that the last term vanishes at the limit. Moreover since in the risk-neutral dynamics (2.11) all coefficients in the matrix $A$ and CIR processes are time independent we conclude that $\xi$ does not depend on $t$. We will define:

$$
\xi(r, \lambda, X):=K \mathbb{E}\left[\int_{0}^{\infty}\left(r_{s}+\overline{\rho_{0}}\right) e^{-\int_{0}^{s}\left(r_{u}+l_{u}+\lambda_{u}\right) d u} d s \mid r_{0}=r, \lambda_{0}=\lambda, X_{0}=X\right](2 .
$$

Note that this implies that $\xi\left(r, \lambda, e_{k}\right)$ is $C^{\infty}$ in the neighborhood of any $(r, \lambda)$, $r>0, \lambda>0$ and for any $k=1, \ldots, N$, see Appendix B for details.

The margin $\overline{\rho_{0}}$ is set to satisfy the equilibrium equation

$$
\xi\left(\overline{r_{0}}, \overline{\lambda_{0}}, \overline{X_{0}}\right)=K
$$

or equivalently

$$
\overline{\rho_{0}}=\frac{1-\mathbb{E}\left[\int_{0}^{\infty} r_{s} e^{-\int_{0}^{s}\left(r_{u}+l_{u}+\lambda_{u}\right) d u} d s \mid r_{0}=\overline{r_{0}}, \lambda_{0}=\overline{\lambda_{0}}, X_{0}=\overline{X_{0}}\right]}{\mathbb{E}\left[\int_{0}^{\infty} e^{-\int_{0}^{s}\left(r_{u}+l_{u}+\lambda_{u}\right) d u} d s \mid r_{0}=\overline{r_{0}}, \lambda_{0}=\overline{\lambda_{0}}, X_{0}=\overline{X_{0}}\right]}>0 .
$$

The last inequality is obtained from:

$$
\begin{aligned}
& \mathbb{E}\left[\int_{0}^{\infty} r_{s} e^{-\int_{0}^{s}\left(r_{u}+l_{u}+\lambda_{u}\right) d u} d s \mid r_{0}=\overline{r_{0}}, \lambda_{0}=\overline{\lambda_{0}}, X_{0}=\overline{X_{0}}\right] \\
& <\mathbb{E}\left[\int_{0}^{\infty} r_{s} e^{-\int_{0}^{s} r_{u} d u} d s \mid r_{0}=\overline{r_{0}}, \lambda_{0}=\overline{\lambda_{0}}, X_{0}=\overline{X_{0}}\right]=1 .
\end{aligned}
$$

Similar arguments show that (see Appendix B)

$$
\begin{aligned}
& \left.\xi(r, \lambda, X) \in] 0, K\left(1+\overline{\rho_{0}}\right)\right], \forall r>0, \lambda>0, X \in E, \\
& \lim _{\|(r, \lambda)\| \rightarrow \infty} \xi\left(r, \lambda, e_{k}\right)=0, \forall k=1, \ldots, N .
\end{aligned}
$$

The above results and the regularity of $\xi$ show that $\xi$ can be extended by continuity when $r=0$ or $\lambda=0$. 
REMARK 2. If an additional commercial margin $\nu_{0}$ is considered then $\overline{\rho_{0}}$ is first computed as above and then replaced by $\overline{\overline{\rho_{0}}}=\overline{\rho_{0}}+\nu_{0}$ in Equation (2.14). With these changes all results of the paper remain valid.

We also introduce for technical reasons the curves $\bar{\Gamma}_{k}^{0}, k=1, \ldots, N$ :

$$
\bar{\Gamma}_{k}^{0}=\left\{(r, \lambda) \mid r \geq 0, \lambda \geq 0, \xi\left(r, \lambda, e_{k}\right)=K\right\} .
$$

Of course, $\left(\overline{r_{0}}, \overline{\lambda_{0}}\right) \in \bar{\Gamma}_{\overline{X_{0}}}^{0}$. We also define the domains:

$$
\begin{aligned}
& \Omega_{k}^{\xi-}=\left\{(r, \lambda) \mid r \geq 0, \lambda \geq 0, \xi\left(r, \lambda, e_{k}\right)<K\right\}, \\
& \Omega_{k}^{\xi+}=\left\{(r, \lambda) \mid r \geq 0, \lambda \geq 0, \xi\left(r, \lambda, e_{k}\right)>K\right\} .
\end{aligned}
$$

2.3. Further properties of the PVRP $\xi$. It is useful for the following to introduce a PDE formulation for $\xi$. To ease the notations we introduce the operator $\mathcal{A}^{\mathcal{R}}$ that acts on regular functions $v(r, \lambda, X)$ as follows:

$\left(\mathcal{A}^{\mathcal{R}} v\right)\left(r, \lambda, e_{k}\right)=\left(\mathcal{A}_{k} v\right)\left(r, \lambda, e_{k}\right)-\left(r+l_{k}+\lambda\right) v\left(r, \lambda, e_{k}\right)+\sum_{j=1}^{N} a_{k, j}\left(v\left(r, \lambda, e_{j}\right)-v\left(r, \lambda, e_{k}\right)\right)$,

where $\mathcal{A}_{k}$ is the characteristic operator (cf. [26, Chapter 7.5]) of the CIR processes of $r$ and $\lambda$ in $X=e_{k}$, i.e., the operator that acts on any $C^{2}$ class function $v(r, \lambda)$ by

$$
\begin{aligned}
\mathcal{A}_{k}(v)(r, \lambda)= & \gamma_{\lambda, k}\left(\theta_{\lambda, k}-\lambda\right) \partial_{\lambda} v(r, \lambda)+\frac{1}{2} \sigma_{\lambda, k}^{2} \lambda \partial_{\lambda \lambda} v(r, \lambda) \\
& +\gamma_{r, k}\left(\theta_{r, k}-r\right) \partial_{r} v(r, \lambda)+\frac{1}{2} \sigma_{r, k}^{2} r \partial_{r r} v(r, \lambda) \\
& +\rho \sqrt{r \lambda} \sigma_{\lambda, k} \sigma_{r, k} \partial_{r \lambda} v(r, \lambda) .
\end{aligned}
$$

Since $\xi$ is regular one can use an adapted version of the Feynman-Kac formula in order to conclude that $\xi$ defined by (2.14) satisfies the equation:

$$
\left(\mathcal{A}^{\mathcal{R}} \xi\right)(r, \lambda, X)+\left(r+\overline{\rho_{0}}\right) K=0, \forall r>0, \lambda>0, \forall X \in E .
$$

2.4. Valuation of the prepayment option. The valuation problem of the prepayment option can be modeled as an American call option (on a risky debt owned by the borrower) with payoff:

$$
\chi(r, \lambda, X)=(\xi(r, \lambda, X)-K)^{+} .
$$

Here the prepayment option allows borrower to buy back and refinance its debt according to the current contractual margin at any time during the life of the option. We denote by $P$ the price of the prepayment option.

General results that have been derived for the pricing of a perpetual (vanilla) American put option [19, 2] show that the stopping time has a simple structure: a critical frontier splits the domain into two regions: the exercise region where it is optimal to exercise and where the price equals the payoff and a continuation region where the price satisfies a partial differential equation similar to the Black-Scholes PDE. We refer to [9] for how to adapt the theoretical arguments for the situation when the dynamics is not Black-Scholes but a CIR process.

The result builds heavily on the geometric properties (convexity, etc.) of the payoff, which are not available in this setting; a direct proof is therefore needed. Note 
that for general payoffs examples are available (see for instance [11]) where several (connected) exercise and / or continuation regions exist. It is therefore not clear $a$ priori what is the geometry of the exercise regions. We prove here a result that allows to certify that, under some technical assumptions given below, for the prepayment option at most one connected exercise region and at most one connected continuation region exist in any regime.

THEOREM 3. Let $\Omega:=\left(\Omega_{k}\right)_{k=1}^{N}$ be a $N$-tuple of connected open sets $\Omega_{k} \subset\left(\mathbb{R}_{+}\right)^{2}$ with piecewise Lipschitz frontiers. Denote by $\Omega_{k}^{c}$ the interior of $\left(\mathbb{R}_{+}\right)^{2} \backslash \Omega_{k}$ and $\Gamma_{k}$ the common frontier of $\Omega_{k}^{c}$ and $\Omega_{k}$. Introduce the function $P_{\Omega}(r, \lambda, X)$ defined by:

$$
\begin{aligned}
& P_{\Omega}\left(r, \lambda, e_{k}\right)=\chi\left(r, \lambda, e_{k}\right) \forall(r, \lambda) \in \Omega_{k}, k=1, \ldots, N \\
& \left(\mathcal{A}^{\mathcal{R}} P_{\Omega}\right)\left(r, \lambda, e_{k}\right)=0, \forall(r, \lambda) \in \Omega_{k}^{c}, k=1, \ldots, N \\
& P_{\Omega}\left(r, \lambda, e_{k}\right)=\chi\left(r, \lambda, e_{k}\right), \text { on } \Gamma_{k}, k=1, \ldots, N \\
& \quad \lim _{\|(r, \lambda)\| \rightarrow \infty} P_{\Omega}\left(r, \lambda, e_{k}\right)=0, k=1, \ldots, N .
\end{aligned}
$$

Suppose $\Omega^{*}:=\left(\Omega_{k}^{*}\right)_{k=1}^{N}$ exists such that for all $k=1, \ldots, N$ the frontier of $\Omega_{k}^{*}$ is piecewise Lipschitz and:

$$
\begin{aligned}
& \Omega_{k}^{*} \subset \Omega_{k}^{\xi+} \\
& P_{\Omega^{*}}(r, \lambda, X) \geq \chi(r, \lambda, X) \forall r, \lambda, X \\
& P_{\Omega^{*}}\left(r, \lambda, e_{k}\right) \text { is of } C^{1} \text { class on }\left(\mathbb{R}_{+}\right)^{2}, k=1, \ldots, N \\
& \sum_{j=1}^{N} a_{k, j}\left(P_{\Omega^{*}}\left(r, \lambda, e_{j}\right)-\chi\left(r, \lambda, e_{j}\right)\right)+K\left(\lambda+l_{k}-\overline{\rho_{0}}\right) \leq 0 \forall(r, \lambda) \in \Omega_{k}^{*} .
\end{aligned}
$$

Then $P=P_{\Omega^{*}}$.

Proof. Denote by $\mathcal{T}$ the ensemble of (positive) stopping times; then for all $k=$ $1, \ldots, N$ :

$$
P\left(r, \lambda, e_{k}\right)=\sup _{\tau \in \mathcal{T}} \mathbb{E}\left[e^{-\int_{0}^{\tau}\left(r_{u}+l_{u}+\lambda_{u}\right) d u} \chi\left(r_{\tau}, \lambda_{\tau}, X_{\tau}\right) \mid r_{0}=r, \lambda_{0}=\lambda, X_{0}=e_{k}\right] .
$$

We note that if $\tau_{\Omega}$ is the stopping time that stops upon exiting the domain $\Omega_{k}^{c}$ when $X=e_{k}$ then for all $\ell=1, \ldots, N$ :

$$
P_{\Omega}\left(r, \lambda, e_{\ell}\right)=\mathbb{E}\left[e^{-\int_{0}^{\tau_{\Omega}} r_{u}+l_{u}+\lambda_{u} d u} \chi\left(r_{\tau_{\Omega}}, \lambda_{\tau_{\Omega}}, X_{\tau_{\Omega}}\right) \mid r_{0}=r, \lambda_{0}=\lambda, X_{0}=e_{\ell}\right]
$$

Note that the stopping time $\tau_{\Omega}$ is finite a.e. and $P_{\Omega}\left(\cdot, \cdot, e_{k}\right)$ is $C^{2}$ except possibly the negligible set $\cup_{k=1}^{N} \partial \Omega_{k}$. Thus $P \geq P_{\Omega}$; when $\Omega$ touches one of the axis $r=0$ or $\lambda=0$ the continuity with respect to $\Omega$ ensured by the boundary condition (2.26) shows that we still have $P \geq P_{\Omega}$. In particular for $\Omega^{*}$ we obtain $P \geq P_{\Omega^{*}}$; all that remains to be proved is the reverse inequality i.e. $P \leq P_{\Omega^{*}}$.

To this end we use a similar technique as in Theorem 10.4.1 [26, Section 10.4 page 227] (see also [34] for similar considerations). First one can invoke the same arguments as in cited reference (cf. Appendix D for technicalities) and work as if $P_{\Omega^{*}}$ is $C^{2}$ (not only $C^{1}$ as the hypothesis ensures).

The Lemma 2.1 below shows that $\mathcal{A}^{\mathcal{R}} P_{\Omega^{*}} \leq 0$ pointwise almost everywhere and is null on $\Omega_{k}^{*, c}$ when $X=e_{k}$. The Ito formula gives

$$
\begin{aligned}
\left.d\left(e^{-\int_{0}^{t}\left(r_{s}+l_{s}+\lambda_{s}\right) d s} P_{\Omega^{*}}\left(r_{t}, \lambda_{t}, X_{t}\right)\right)\right)= & \left.e^{-\int_{0}^{t}\left(r_{s}+l_{s}+\lambda_{s}\right) d s}\left(\mathcal{A}^{\mathcal{R}} P_{\Omega^{*}}\right)\left(r_{t}, \lambda_{t}, X_{t}\right)\right) d t \\
& +d(\text { martingale })
\end{aligned}
$$


Taking averages and integrating from 0 to some stopping time $\tau$ it follows from $\mathcal{A}^{\mathcal{R}} P_{\Omega^{*}} \leq 0$ that

$$
\begin{aligned}
& P_{\Omega^{*}}(r, \lambda, X) \geq \mathbb{E}\left[e^{-\int_{0}^{\tau}\left(r_{u}+l_{u}+\lambda_{u}\right) d u} P_{\Omega^{*}}\left(r_{\tau}, \lambda_{\tau}, X_{\tau}\right) \mid r_{0}=r, \lambda_{0}=\lambda, X_{0}=X\right] \\
& \geq \mathbb{E}\left[e^{-\int_{0}^{\tau}\left(r_{u}+l_{u}+\lambda_{u}\right) d u} \chi\left(r_{\tau}, \lambda_{\tau}, X_{\tau}\right) \mid r_{0}=r, \lambda_{0}=\lambda, X_{0}=X\right] .
\end{aligned}
$$

Since this is true for any stopping time $\tau$ the conclusion follows. $\square$

LEMma 2.1. Under the hypothesis of the Theorem 3 the following inequality holds pointwise almost everywhere on $\mathbb{R}_{+}^{2}$ :

$$
\left(\mathcal{A}^{\mathcal{R}} P_{\Omega^{*}}\right)(r, \lambda, X) \leq 0, \forall r, \lambda>0, \forall X .
$$

Proof. From (2.27) the conclusion is trivially verified for $X=e_{k}$ for any $(r, \lambda) \in$ $\Omega_{k}^{* c}$.

The non-trivial part of this lemma comes from the fact that if for fixed $k, r_{1}>0$, $\lambda_{1}>0$

$$
P_{\Omega^{*}}\left(r, \lambda, e_{k}\right)=\chi\left(r, \lambda, e_{k}\right)
$$

for any $(r, \lambda)$ in some the neighborhood of $\left(r_{1}, \lambda_{1}\right)$ this does not necessarily imply

$$
\left(\mathcal{A}^{\mathcal{R}} P_{\Omega^{*}}\right)\left(r_{1}, \lambda_{1}, e_{k}\right)=\left(\mathcal{A}^{\mathcal{R}} \chi\right)\left(r_{1}, \lambda_{1}, e_{k}\right)
$$

because $\mathcal{A}^{\mathcal{R}}$ depends on other values $P_{\Omega^{*}}\left(r, \lambda, e_{j}\right)$ with $j \neq k$.

Suppose now $(r, \lambda) \in \cap_{j=1}^{N} \Omega_{j}^{*}$; this means in particular that $\Omega_{k}^{*} \neq \emptyset$ and from hypothesis (2.30) also $\Omega_{k}^{\xi+} \neq \emptyset$ and moreover $\xi\left(r, \lambda, e_{k}\right)>K$ for any $k=1, \ldots, N$; thus $\chi\left(r, \lambda, e_{k}\right)=\xi\left(r, \lambda, e_{k}\right)-K$ for any $k$. Furthermore since $(r, \lambda) \in \cap_{j=1}^{N} \Omega_{j}^{*}$ we have $P_{\Omega^{*}}\left(r, \lambda, e_{k}\right)=\chi\left(r, \lambda, e_{k}\right)=\xi\left(r, \lambda, e_{k}\right)-K$ for any $k$. Fix $X=e_{k}$; then

$$
\begin{aligned}
& \left(\mathcal{A}^{\mathcal{R}} P_{\Omega^{*}}\right)\left(r, \lambda, e_{k}\right)=\left(\mathcal{A}^{\mathcal{R}} \chi\right)\left(r, \lambda, e_{k}\right)=\left(\mathcal{A}^{\mathcal{R}}(\xi-K)\right)\left(r, \lambda, e_{k}\right)=\left(\mathcal{A}^{\mathcal{R}} \xi\right)\left(r, \lambda, e_{k}\right) \\
& -\mathcal{A}^{\mathcal{R}}(K)=-\left(r+\overline{\rho_{0}}\right) K+\left(r+l_{k}+\lambda\right) K=K\left(l_{k}+\lambda-\overline{\rho_{0}}\right) \leq 0,
\end{aligned}
$$

the last inequality being true by hypothesis.

A last situation is when $\lambda \in \Omega_{k}^{*} \backslash \cap_{j=1}^{N} \Omega_{j}^{*}$; there $P_{\Omega^{*}}\left(r, \lambda, e_{k}\right)=\chi\left(r, \lambda, e_{k}\right)$ but some terms $P_{\Omega^{*}}\left(r, \lambda, e_{j}\right)$ for $j \neq k$ may differ from $\chi\left(r, \lambda, e_{j}\right)$. The computation is more technical in this case. This point is specific to the fact that the payoff $\chi$ itself has a complex structure and as such was not emphasized in previous works (e.g., [34], etc.).

Recalling the properties of $\xi$ one obtains using $P_{\Omega^{*}}\left(r, \lambda, e_{k}\right)=\chi\left(r, \lambda, e_{k}\right)$ :

$$
\begin{aligned}
& \left(\mathcal{A}^{\mathcal{R}} P_{\Omega^{*}}\right)\left(r, \lambda, e_{k}\right)=\left(\mathcal{A}_{k} \chi\right)\left(r, \lambda, e_{k}\right)-\left(r+l_{k}+\lambda\right) \chi\left(r, \lambda, e_{k}\right) \\
& +\sum_{j=1}^{N} a_{k, j}\left(P_{\Omega^{*}}\left(r, \lambda, e_{j}\right)-\chi\left(r, \lambda, e_{k}\right)\right) \\
& =\left(\mathcal{A}^{\mathcal{R}} \chi\right)\left(r, \lambda, e_{k}\right)+\sum_{j=1}^{N} a_{k, j}\left(P_{\Omega^{*}}\left(r, \lambda, e_{j}\right)-\chi\left(r, \lambda, e_{j}\right)\right) \\
& =\left(\mathcal{A}^{\mathcal{R}} \xi\right)\left(r, \lambda, e_{k}\right)-\mathcal{A}^{\mathcal{R}}(K)+\sum_{j=1}^{N} a_{k, j}\left(P_{\Omega^{*}}\left(r, \lambda, e_{j}\right)-\chi\left(r, \lambda, e_{j}\right)\right) \\
& =-K\left(r+\overline{\rho_{0}}\right)+\left(r+l_{k}+\lambda\right) K+\sum_{j=1}^{N} a_{k, j}\left(P_{\Omega^{*}}\left(r, \lambda, e_{j}\right)-\chi\left(r, \lambda, e_{j}\right)\right) \leq 0,(2.3
\end{aligned}
$$


where for the last inequality we use hypothesis (2.33). Finally, since we proved that $\left(\mathcal{A}^{\mathcal{R}} P_{\Omega^{*}}\right)(r, \lambda, X) \leq 0$ strongly except for some values depending on the frontiers of $\Omega_{k}^{*}$ and since $P_{\Omega^{*}}$ is of $C^{1}$ class we obtain the conclusion. $\square$

REMARK 4. Several remarks are in order at this point:

1. when $N>1$ checking (2.33) does not involve any computation of derivatives and is straightforward.

2. as mentioned in the previous section, the Theorem is a verification result i.e., only gives sufficient conditions for a candidate to be the option price.

3. the candidate solution $\Omega^{*}$ can be found either by maximizing the function $\Omega \mapsto P_{\Omega}\left(\overline{r_{0}}, \overline{\lambda_{0}}, \overline{X_{0}}\right)$ with respect to all admissible $\Omega$ (which is difficult for 2-dimensional domains) or by solving a constraint optimization problem as seen below.

3. A minimization problem and the numerical algorithm. The Theorem 3 is a verification result. Its utility is to guarantee that a candidate $\Omega^{*}$ is solution once such a candidate is found. But the Theorem does not say how to find $\Omega^{*}$. To this end we rewrite our problem in a different framework, that of a minimization problem based on a variational inequality as explained below. It is worth mentioning that variational inequalities are naturally associated to an American option but the nonstandard payoff here does not allow to obtain information on the geometry of the exercise and continuation regions directly from classic approaches to such variational inequalities. We refer the reader to [21] for further information on the mathematical objects involved.

The results of this Section are proved under the assumption that the Markov chain $X_{t}$ has a stationary distribution. This assumption is not restrictive in practice. In conjunction with the existence of a stationary distribution for each CIR process it allows to consider the joint stationary distribution of the dynamics $\left(r_{t}, \lambda_{t}, X_{t}\right)$, whose density is denoted $\mu(r, \lambda, X)$. To ease notations when there is no ambiguity we write $\mu_{k}$ or $\mu_{k}(r, \lambda)$ instead of $\mu\left(r, \lambda, e_{k}\right)$. Note that since $\mu$ represents a probability density it is always (strictly) positive. Moreover, see Appendix C, one can prove that $\mu_{k}$ is $C^{\infty}$ and all moments are finite.

Introduce for functions $u, v: \mathbb{R}_{+} \times \mathbb{R}_{+} \times E \rightarrow \mathbb{R}$ (with $u_{k}:=u\left(\cdot, \cdot, e_{k}\right)$ and same for $v$ ) the notation:

$$
\begin{aligned}
& \langle u, v\rangle_{\star}=\sum_{k=1}^{N} \iint_{\left(\mathbb{R}_{+}\right)^{2}}\left\{\frac{\sigma_{\lambda, k}^{2}}{2} \lambda \partial_{\lambda} u_{k} \partial_{\lambda} v_{k}+\frac{\sigma_{r, k}^{2}}{2} r \partial_{r} u_{k} \partial_{r} v_{k}+\right. \\
& \left.\rho \sigma_{r, k} \sigma_{\lambda, k} \sqrt{r \lambda} \frac{\partial_{r} u_{k} \partial_{\lambda} v_{k}+\partial_{r} v_{k} \partial_{\lambda} u_{k}}{2}+u_{k} v_{k}\left(r+\lambda+l_{k}\right)\right\} \mu_{k} d r d \lambda \\
& +\int_{0}^{\infty}\left(\gamma_{\lambda, k} \theta_{\lambda, k}-\frac{\sigma_{\lambda, k}^{2}}{2}\right) \frac{u_{k}(r, 0) v_{k}(r, 0)}{2} \mu_{k}(r, 0) d r \\
& +\int_{0}^{\infty}\left(\gamma_{r, k} \theta_{r, k}-\frac{\sigma_{r, k}^{2}}{2}\right) \frac{u_{k}(0, \lambda) v_{k}(0, \lambda)}{2} \mu_{k}(0, \lambda) d \lambda+ \\
& +\sum_{1 \leq j<k \leq N} \iint_{\left(\mathbb{R}_{+}\right)^{2}} \frac{\left(a_{j, k} \mu_{j}+a_{k, j} \mu_{k}\right)\left(u_{k}-u_{j}\right)\left(v_{k}-v_{j}\right)}{2} d r d \lambda,
\end{aligned}
$$

and denote by $\mathcal{H}_{\star}$ the space:

$$
\mathcal{H}_{\star}=\left\{u: \mathbb{R}_{+} \times \mathbb{R}_{+} \times E \rightarrow \mathbb{R} \mid\langle u, u\rangle_{\star}<\infty\right\} .
$$


Define also for smooth functions the bilinear form

$$
a_{\star}(u, v)=\sum_{k=1}^{N} \iint_{\left(\mathbb{R}_{+}\right)^{2}}\left(-\mathcal{A}^{\mathcal{R}}(u)\right)\left(r, \lambda, e_{k}\right) v\left(r, \lambda, e_{k}\right) \mu_{k} d r d \lambda .
$$

The difficulty of proposing a variational inequality framework is to come up with the adequate function spaces and exhibit convenient properties of the bi-linear form $a_{\star}$. The good news, not a priori guaranteed, is that it is possible to find a functional space (space $\mathcal{H}_{\star}$ ) where the variational inequality is equivalent to a minimization problem; the space $\mathcal{H}_{\star}$ is a weighted Sobolev space (non-weighted spaces were also considered but do not have convenient properties).

TheOrem 5. Suppose that the Markov chain with transition matrix A admits a stationary distribution. Then

1. The space $\mathcal{H}_{\star}$ is a Hilbert space with scalar product $\langle\cdot, \cdot\rangle_{\star}$. We will denote by $\|\cdot\|_{\star}$ its norm.

2. The form $a_{\star}$ admits a unique continuous extension to $\mathcal{H}_{\star} \times \mathcal{H}_{\star}$ (denoted still $\left.a_{\star}\right), \chi \in \mathcal{H}_{\star}$ and the problem

$$
\min \left\{a_{\star}(u, u-\chi) \mid u \in \mathcal{H}_{\star}, u \geq \chi, a_{\star}(u, v) \geq 0, \forall v \in \mathcal{H}_{\star}, v \geq 0\right\},
$$

is well posed and admits a unique solution $U_{\star}$.

3. Consider $\Omega^{*}$ that satisfies the hypothesis of the Theorem 3. Then $P=U_{\star}$.

Proof.

$1 /$ We first prove that $\langle\cdot, \cdot\rangle_{\star}$ is a scalar product. The property to prove is the strict positivity. But since $|\rho| \leq 1$ by Cauchy-Schwartz:

$$
\iint_{\left(\mathbb{R}_{+}\right)^{2}}\left\{\frac{\sigma_{\lambda, k}^{2}}{2} \lambda\left(\partial_{\lambda} u_{k}\right)^{2}+\frac{\sigma_{r, k}^{2}}{2} r\left(\partial_{r} u_{k}\right)^{2}+\rho \sigma_{r, k} \sigma_{\lambda, k} \sqrt{r \lambda} \partial_{r} u_{k} \partial_{\lambda} u_{k}\right\} d r d \lambda \geq 0
$$

Under hypotheses (2.8)-(2.9) the other terms are also positive; moreover the sum of all terms is strictly positive as soon as the function $u$ is non-null.

$2 /$ We prove that for regular enough functions

$$
a_{\star}(u, v)=\langle u, v\rangle_{\star}+b_{\star}(u, v),
$$

where $b_{\star}: \mathcal{H}_{\star} \times \mathcal{H}_{\star} \rightarrow \mathbb{R}$ is a continuous, antisymmetric (i.e., $b_{\star}(u, v)+b_{\star}(v, u)=0$ ) bilinear form.

To this end one has to integrate by parts all terms appearing in the definition of the form $a_{\star}$. We take for instance the correlation term and compute for regular functions $f, g, h$ with exponential decay at infinity (see Appendix A for details):

$$
\begin{aligned}
& \iint_{\left(\mathbb{R}_{+}\right)^{2}} \partial_{r \lambda} f g \sqrt{r \lambda} h d r d \lambda=-\iint_{\left(\mathbb{R}_{+}\right)^{2}} \partial_{\lambda} f \partial_{r} \sqrt{r \lambda} h d r d \lambda+\iint_{\left(\mathbb{R}_{+}\right)^{2}} \frac{f g \partial_{\lambda r}(\sqrt{r \lambda} h)}{2} d r d \lambda \\
& \left.+\iint_{\left(\mathbb{R}_{+}\right)^{2}} \frac{f \partial_{\lambda} g-g \partial_{\lambda} f}{2} \partial_{r}(\sqrt{r \lambda} h)\right) d r d \lambda .
\end{aligned}
$$

The first term enters in the definition of the scalar product and is symmetric; last term is antisymmetric. The middle term will be seen to simplify latter on. This identity will be used for $f=u_{k}, g=v_{k}, h=\mu_{k}$ for all $k$. Similar identities are found for terms involving derivatives. We obtain by summation that $a_{\star}(u, v)$ is the sum of : 
$-\langle u, v\rangle_{\star}$ except the last term,

- an antisymmetric continuous bilinear form

- the quantity: $\sum_{k=1}^{N} \iint_{\left(\mathbb{R}_{+}\right)^{2}} \frac{u_{k} v_{k}}{2}\left(-\mathcal{A}_{k}^{*}\left(\mu_{k}\right)\right)-\sum_{j=1}^{N} a_{k, j}\left(u_{j}-u_{k}\right) v_{k} \mu_{k} d r d \lambda$.

Here $\mathcal{A}_{k}^{*}$ is the adjoint of $\mathcal{A}_{k}^{*}$ and acts on regular functions $w$ by:

$$
\begin{aligned}
\mathcal{A}_{k}^{*}(w)= & -\partial_{\lambda}\left(\gamma_{\lambda, k}\left(\theta_{\lambda, k}-\lambda\right) w\right)+\frac{\sigma_{\lambda, k}^{2}}{2} \partial_{\lambda \lambda}(\lambda w)-\partial_{r}\left(\gamma_{r, k}\left(\theta_{r, k}-r\right) w\right)+\frac{\sigma_{r, k}^{2}}{2} \partial_{r r}(r w) \\
& +\rho \sigma_{\lambda, k} \sigma_{r, k} \partial_{r \lambda}(\sqrt{r \lambda} w) .
\end{aligned}
$$

Note that $\mu$ is solution to the following PDE (of Fokker-Plank / forward Kolmogorov type):

$$
\left(\mathcal{A}_{k}^{*}(\mu)\right)\left(r, \lambda, e_{k}\right)+\sum_{j=1}^{N} a_{j, k} \mu_{j}-a_{k, j} \mu_{k}=0 .
$$

Thus

$$
\begin{aligned}
& \sum_{k=1}^{N} \iint_{\left(\mathbb{R}_{+}\right)^{2}} \frac{u_{k} v_{k}}{2}\left(-\mathcal{A}_{k}^{*}\left(\mu_{k}\right)\right)-\sum_{j=1}^{N} a_{k, j}\left(u_{j}-u_{k}\right) v_{k} \mu_{k} d r d \lambda \\
& =\sum_{k, l=1}^{N} \iint_{\left(\mathbb{R}_{+}\right)^{2}} \frac{u_{k} v_{k}}{2}\left(a_{j, k} \mu_{j}-a_{k, j} \mu_{k}\right)-a_{k, j}\left(u_{j}-u_{k}\right) v_{k} \mu_{k} d r d \lambda \\
& =\sum_{1 \leq j<k \leq N} \iint_{\left(\mathbb{R}_{+}\right)^{2}} \frac{u_{k} v_{k}}{2}\left(a_{j, k} \mu_{j}-a_{k, j} \mu_{k}\right)-a_{k, j}\left(u_{j}-u_{k}\right) v_{k} \mu_{k} d r d \lambda \\
& =\sum_{1 \leq j<k \leq N} \iint_{\left(\mathbb{R}_{+}\right)^{2}} \frac{\left(a_{j, k} \mu_{j}+a_{k, j} \mu_{k}\right)\left(u_{k}-u_{j}\right)\left(v_{k}-v_{j}\right)}{2}+\frac{a_{k, j} \mu_{k}\left(u_{j} v_{k}-u_{k} v_{j}\right)}{2},
\end{aligned}
$$

which provides the last term in the scalar product part and also the continuous antisymmetric bilinear form $\sum_{1 \leq j<k \leq N} \iint_{\left(\mathbb{R}_{+}\right)^{2}} \frac{a_{k, j} \mu_{k}\left(u_{j} v_{k}-u_{k} v_{j}\right)}{2}$. This concludes the proof of (3.6).

Since $b_{\star}$ is continuous $\langle u, v\rangle_{\star}+b_{\star}(u, v)$ is a continuous bilinear form on $\mathcal{H}_{\star} \times \mathcal{H}_{\star}$. Moreover this form equals $a_{\star}$ on a dense subset, thus $a_{\star}$ admits a unique continuous extension to $\mathcal{H}_{\star} \times \mathcal{H}_{\star}$ given by $\langle u, v\rangle_{\star}+b_{\star}(u, v)$. We still denote by $a_{\star}$ this extension.

We prove in Appendix B that $\xi, \chi, P \in \mathcal{H}_{\star}$.

Since $a_{\star}(\cdot, \chi)$ is a continuous linear form on $\mathcal{H}_{\star}$ one can represent it as $\langle\cdot, \zeta\rangle_{\star}$ for some $\zeta \in \mathcal{H}_{\star}$. Then

$$
a_{\star}(u, u-\chi)=a_{\star}(u, u)-a_{\star}(u, \chi)=\langle u, u-\zeta\rangle_{\star} .
$$

Consider $\left(u_{n}\right)_{n \in \mathbb{N}}$ a minimizing sequence for the problem. There exists $M>0$ such that $a_{\star}\left(u_{n}, u_{n}-\chi\right)=\left\langle u_{n}, u_{n}-\zeta\right\rangle_{\star} \leq M$ for all $n$. Thus

$$
\left\|u_{n}\right\|_{\star}^{2}=\left\langle u_{n}, u_{n}\right\rangle_{\star} \leq M+\left\langle u_{n}, \zeta\right\rangle_{\star} \leq M+\frac{\left\|u_{n}\right\|_{\star}^{2}+\|\zeta\|_{\star}^{2}}{2},
$$

which shows that $\left\|u_{n}\right\|_{\star}^{2}$ is bounded. Up to extracting a subsequence one can assume that $\left(u_{n}\right)_{n}$ is weakly convergent to some $U_{\star}$. Taking into account the norm of the space, the convergence is strong $L_{l o c}^{2}$. In particular from $u_{n} \geq \chi$ it follows $U_{\star} \geq \chi$. 
Consider now $v \in \mathcal{H}_{\star}$ with $v \geq 0$. Then from $0 \leq a_{\star}\left(u_{n}, v\right)$ and (by weak convergence) $\lim _{n \rightarrow \infty} a_{\star}\left(u_{n}, v\right)=a_{\star}\left(U_{\star}, v\right)$ one concludes that $a_{\star}\left(U_{\star}, v\right) \geq 0$ i.e., $U_{\star}$ is admissible.

Note also that weak convergence implies

$$
a_{\star}\left(U_{\star}, U_{\star}\right)=\left\|U_{\star}\right\|_{\star}^{2} \leq \liminf _{n \rightarrow \infty}\left\|u_{n}\right\|_{\star}^{2}=\liminf _{n \rightarrow \infty} a_{\star}\left(u_{n}, u_{n}\right) .
$$

Since $\lim _{n \rightarrow \infty} a_{\star}\left(u_{n}, \chi\right)=a_{\star}\left(U_{\star}, \chi\right)$ one obtains

$$
a_{\star}\left(U_{\star}, U_{\star}-\chi\right) \leq \liminf _{n \rightarrow \infty} a_{\star}\left(u_{n}, u_{n}-\chi\right),
$$

thus $U_{\star}$ is a minimizer and $U_{\star} \in \mathcal{H}_{\star}$.

Suppose now that there exist two minimizers $U_{\star}^{1}$ and $U_{\star}^{2}$. Denote

$$
m=a_{\star}\left(U_{\star}^{1}, U_{\star}^{1}-\chi\right)=a_{\star}\left(U_{\star}^{2}, U_{\star}^{2}-\chi\right) .
$$

Then one notes that $\frac{U_{\star}^{1}+U_{\star}^{2}}{2}$ is an admissible point. Moreover, from minimality

$$
\begin{aligned}
& m \leq a_{\star}\left(\frac{U_{\star}^{1}+U_{\star}^{2}}{2}, \frac{U_{\star}^{1}+U_{\star}^{2}}{2}-\chi\right)=\left\|\frac{U_{\star}^{1}+U_{\star}^{2}}{2}\right\|_{\star}^{2}-a_{\star}\left(\frac{U_{\star}^{1}+U_{\star}^{2}}{2}, \chi\right) \\
& =\left\|\frac{U_{\star}^{1}+U_{\star}^{2}}{2}\right\|_{\star}^{2}-\frac{\left\|U_{\star}^{1}\right\|_{\star}^{2}+\left\|U_{\star}^{2}\right\|_{\star}^{2}}{2}+m=m-\left\|\frac{U_{\star}^{1}-U_{\star}^{2}}{2}\right\|_{\star}^{2},
\end{aligned}
$$

which implies $U_{\star}^{1}=U_{\star}^{2}$.

$3 /$ We proved that $-\mathcal{A}^{\mathcal{R}} P \geq 0$ (except possibly a null measure set). Thus when one multiplies by any positive, sufficiently smooth, function $v$ one obtains after integration

$$
\sum_{k=1}^{N} \iint_{\left(\mathbb{R}_{+}\right)^{2} \times\left\{e_{k}\right\}}\left(-\mathcal{A}^{\mathcal{R}} P\right) v \mu_{k} \geq 0,
$$

i.e., $a_{\star}(P, v) \geq 0$. By density the result will be true for any positive $v \in \mathcal{H}_{\star}$. Recalling that $P \geq \chi$ one obtains that $P$ is an admissible function for the minimization (3.4). Moreover $a_{\star}(P, P-\chi)=0 \leq a_{\star}(u, u-\chi)$ for any admissible $u$ (take $\left.v=u-\chi\right)$, hence the conclusion. $\square$

REMARK 6.

1. The result above is used in the numerical implementation to find a "solution candidate". This candidate is then validated through verification result in the Theorem 3; the candidate is obtained by solving a linear constrained quadratic optimization problem with strictly positive Hessian that is obtained from suitable discretization of the bi-linear form $a_{\star}$; the discretization of the form $a_{\star}$ is explained in the next section. However the reader should not be mislead by the conceptually "simple" framework of a quadratic optimization problem under convex constraints: convex optimization problems may be numerically very time consuming when the number of constraints is high, as is the situation here. Numerical algorithms that address this problem are however available, cf. [4].

2. Once a "solution candidate" is found it has to satisfy all hypothesis of Theorem 3, in particular the hypothesis (2.32). Note that since the candidate 
is $C^{2}$ on domains $\Omega_{k}^{*}$ and $\Omega_{k}^{* c}$ only the conditions on the frontier $\Gamma_{k}^{*}$ is to be satisfied. The continuity is straightforward to check. For the continuity of the derivatives one notes that only the continuity of the normal derivative (the normal is with respect to the frontier $\Gamma_{k}^{*}$ ) is to be verified: for all other directions the derivative will be continuous because is the trace on $\Gamma_{k}^{*}$ of the derivative of a $C^{2}$ function.

4. Numerical Application. In order to discretize the bi-linear form $a_{\star}$ (see Remark 6) we have to propose a discretization for the operators $\mathcal{A}^{\mathcal{R}}$ and $\mathcal{A}_{k}^{*}$. We used two different numerical implementations and both give similar results.

One implementation uses a finite difference method (written in $M A T L A B^{\circledR}$ ) based on a grid with the time step $\Delta r$ and space step $\Delta \lambda$ and look for an approximation $P_{n, \ell, k}$ of $P_{\Omega^{*}}\left(n \Delta r, \ell \Delta \lambda, e_{k}\right)$. The domain is truncated at $\lambda_{\max }$ and $r_{\max }$. We choose $\lambda_{\max }=6000 b p s, r_{\max }=2000 b p s$. Recall that a basis point, denoted "1 bps" equals $10^{-4}$.

The first and second derivatives are approximated by (centered) finite difference formula. We obtain for instance the discretization of $\mathcal{A}^{\mathcal{R}}(P)$ :

$$
\begin{aligned}
& \gamma_{r, k}\left(\theta_{r, k}-(n \Delta r)\right) \frac{P_{n+1, \ell, k}-P_{n-1, \ell, k}}{2 \Delta r}+\frac{\sigma_{r, k}^{2}}{2}(n \Delta r) \frac{P_{n+1, \ell, k}-2 P_{n, \ell, k}+P_{n-1, \ell, k}}{\Delta r^{2}} \\
& +\gamma_{\lambda, k}\left(\theta_{\lambda, k}-(\ell \Delta r)\right) \frac{P_{n, \ell+1, k}-P_{n, \ell-1, k}}{2 \Delta \lambda}+\frac{\sigma_{\lambda, k}^{2}}{2}(\ell \Delta \lambda) \frac{P_{n, \ell+1, k}-2 P_{n, \ell, k}+P_{n, \ell-1, k}}{\Delta \lambda^{2}} \\
& +\rho \sigma_{r, k} \sigma_{\lambda, k} \sqrt{n \Delta r \cdot \ell \Delta \lambda} \frac{\left(P_{n+1, \ell+1, k}-P_{n-1, \ell+1, k}\right)-\left(P_{n+1, \ell-1, k}-P_{n-1, \ell-1, k}\right)}{(2 \Delta r)(2 \Delta \lambda)} \\
& -\left((n \Delta r)+l_{k}+(\ell \Delta \lambda)\right) P_{n, \ell, k}+\sum_{j=1}^{N} a_{k, j}\left[P_{n, \ell, j}-P_{n, \ell, k}\right] .
\end{aligned}
$$

Since the bi-linear form is degenerate at the boundaries $r=0, \infty, \lambda=0, \infty$ there is no need to impose boundary conditions at these points. In practice, in order to obtain as many equations as unknowns, for the last point before boundary, e.g., $P_{1, \ell, k}$ we use de-centered finite differences.

A second implementation used the FreeFem ++ software (see [16] for details). This code is very efficient and implements a finite element method. Freefem ++ was used to discretize the operators and obtain the matrix of $a_{\star}$ in a Galerkin basis. Then the optimization was performed with Octave (see [13]).

4.1. Application 1 : 1 regime. We consider a perpetual loan with a nominal $K=1$ and one regime $(N=1)$. We omit in the following the variable $X$ assigned to the regime. The borrower default intensity $\lambda_{t}$ follows a CIR process with parameters: initial intensity $\overline{\lambda_{0}}=100 \mathrm{bps}$, volatility $\sigma_{\lambda}=0.1$, average intensity $\theta_{\lambda}=220 \mathrm{bps}$, reversion coefficient $\gamma_{\lambda}=0.1$. On the interbank market, the CIR process of the LIBOR has the following parameters: initial LIBOR $\overline{r_{0}}=4 \%$, volatility $\sigma_{r}=0.1$, average intensity $\theta_{r}=4.6 \%$, reversion coefficient $\gamma_{r}=0.8$. We assume a unique and constant liquidity cost $l_{1}=50 \mathrm{bps}$.

In order to find the initial contractual margin we use equation (2.15) and find $\overline{\rho_{0}}=196 \mathrm{bps}$. Therefore, we can represent $\xi(r, \lambda)$ according to the current intensity $\lambda$ and LIBOR $r$, see Figure 4.2. We illustrate the dependence of $\xi(r, \lambda)$ around $\left(\overline{r_{0}}, \overline{\lambda_{0}}\right)$ separately with respect to both variables $r, \lambda$ in the Figure 4.1 . Note that $\xi\left(\overline{r_{0}}, \lambda\right)$ is very sensitive to the borrower's credit quality and it decreases when $\lambda$ rises; on the contrary $\xi\left(r, \overline{\lambda_{0}}\right)$ exhibits a low sensitivity to LIBOR variations. 

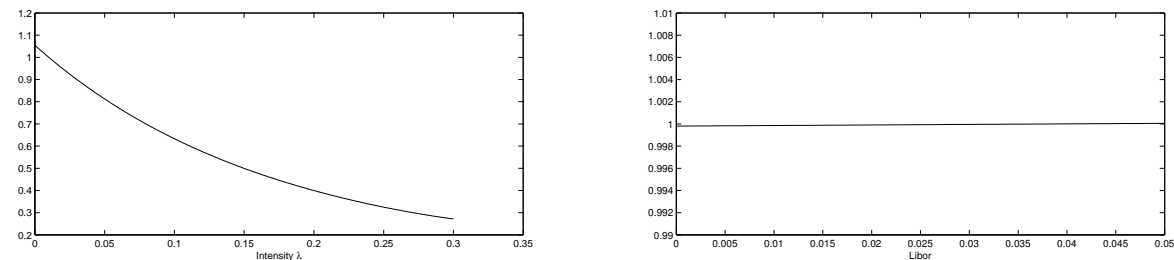

FIG. 4.1. PVRP value as a function of the intensity (left: $\left.\xi\left(\overline{r_{0}}, \lambda\right)\right)$ and LIBOR (right: $\xi\left(r, \overline{\lambda_{0}}\right)$ ) for the inputs in Section 4.1.

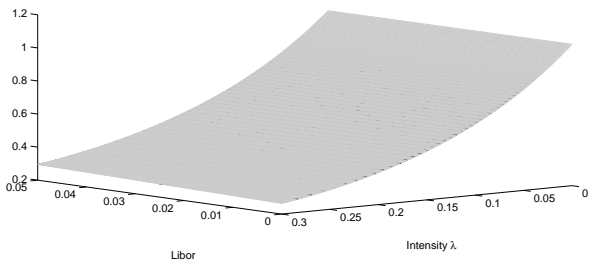

FIG. 4.2. $\xi(r, \lambda)$ for the inputs in Section 4.1. $\xi$ is decreasing when there is a degradation of the credit quality (i.e., $\lambda$ increases) and converges to 0 at infinity.

The price $P$ and the optimal frontier $\Gamma^{*}$ are obtained with the algorithm in Section 3 and are validated by checking the hypothesis of the Theorem 3 ; the optimal frontier defines the exercise region (below the curve) and the continuation region (above the curve), see Figure 4.3. At origination, the present value of cash flows is at par, so $\xi\left(\overline{r_{0}}, \overline{\lambda_{0}}\right)=1$. The prepayment option price is $P\left(\overline{r_{0}}, \overline{\lambda_{0}}\right)=0.0347=3.61 \% \cdot K$, see Figure 4.4. We illustrate the dependence of the option $P(r, \lambda)$ with respect to both variables $(r, \lambda)$ around $\left(\overline{r_{0}}, \overline{\lambda_{0}}\right)$ in the Figure 4.5. Note that $P\left(\overline{r_{0}}, \lambda\right)$ is very sensitive to the borrower's credit quality $\lambda$ and it decreases when $\lambda$ rises. On the contrary $P\left(r, \overline{\lambda_{0}}\right)$ exhibits a low sensitivity with respect to LIBOR variations.

Therefore the loan value equals $\xi\left(\overline{r_{0}}, \overline{\lambda_{0}}\right)-P\left(\overline{r_{0}}, \overline{\lambda_{0}}\right)=0,9653$. If the bank decides to include the prepayment option in the initial contractual margin $\overline{\overline{\rho_{0}}}$ (cf. Remark 2) then $\overline{\overline{\rho_{0}}}=346 b p s$. Note that $\overline{\overline{\rho_{0}}}$ is significantly higher than $\overline{\rho_{0}}$.

4.2. Application $2: 2$ regimes. Consider a loan with a nominal $K=1$ in an environment with two economic states: state $e_{1}$ corresponds to economic expansion and state $e_{2}$ to a recession. The borrower default intensity $\lambda_{t}$ follows a CIR process with different parameters according to the economic state: initial intensity $\overline{\lambda_{0}}=212 \mathrm{bps}$, volatility $\left(\sigma_{\lambda, 1}, \sigma_{\lambda, 2}\right)=(0.1,0.2)$, average intensity $\left(\theta_{\lambda, 1}, \theta_{\lambda, 2}\right)=$ (220bps, 1680bps), reversion coefficient $\left(\gamma_{\lambda, 1}, \gamma_{\lambda, 2}\right)=(0.1,0.2)$. The default intensity process reflects a higher credit risk in state $e_{2}$.

The CIR process of the LIBOR is defined with the following parameters: initial LIBOR $\overline{r_{0}}=4 \%$, volatility $\left(\sigma_{r, 1}, \sigma_{r, 2}\right)=(0.1,0.01)$, average intensity $\left(\theta_{r, 1}, \theta_{r, 2}\right)=$ $(4.6 \%, 0.3 \%)$, reversion coefficient $\left(\gamma_{r, 1}, \gamma_{r, 2}\right)=(0.8,0.3)$. We take the correlation $\rho$ to be null. The LIBOR is linked to the Central Bank rates: during a state of economic expansion, the Central Bank rises the rates to avoid inflation and during a recession, the Central Bank decreases the rates to help economic growth. Of course the mathematical model can accommodate any other Central Bank policy.

We assume a liquidity cost defined by a Markov chain of two states $l_{1}=0 b p s$ 


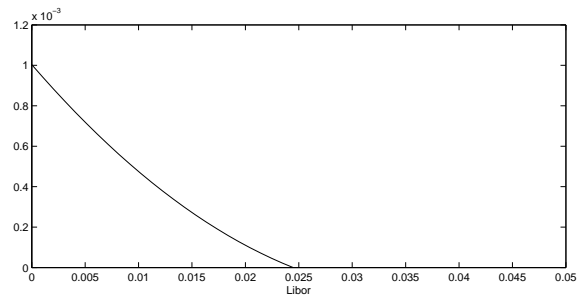

FIG. 4.3. The optimal boundary function $\Gamma^{*}$ as function of the LIBOR $r$ for the inputs in Section 4.1. Two regions appear: the continuation region (above the curve) and the exercise region (below the curve).

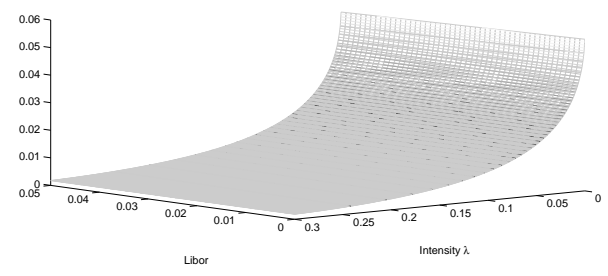

Fig. 4.4. The price $P(r, \lambda)$ for the inputs in Section 4.1.

and $l_{2}=290 \mathrm{bps}$. For $N=2$ the rate $A$ matrix is completely defined by $\alpha_{1,2}=1 / 5$, $\alpha_{2,1}=1 / 5$.

In order to find the initial contractual margin we use equation (2.15) and find $\overline{\rho_{0}}=851 \mathrm{bps}$ in the state $e_{2}$. The contractual margin takes into account the credit risk (default intensity) and the liquidity cost. In this situation $\xi\left(r, \lambda, e_{1}\right)$ is higher than $\xi\left(r, \lambda, e_{2}\right)$ according to the degradation of the credit quality, through the intensity process parameters, and the degradation of the access to money market involving an increase of the funding costs $l_{k}$, see Figures 4.6.

The optimal frontiers $\Gamma_{1}^{*}$ and $\Gamma_{2}^{*}$ are obtained with the algorithm in Section 3 and are validated by checking the hypothesis of the Theorem 3; we obtain $\left(\overline{r_{0}}, 340 b p s\right) \in$ $\Gamma_{1}^{*},\left(\overline{r_{0}}, 0\right) \in \Gamma_{2}^{*}$, see Figure 4.7. Both frontiers delimit the exercise region (below the curve) and the continuation region (above the curve). In state $e_{2}$, the optimal frontier is at 0 for all $r$, because in this particular case it is never optimal to prepay.

We illustrate the dependence of the option $P\left(r, \lambda, \overline{X_{0}}\right)$ with respect to both variables $(r, \lambda)$ in the Figures 4.8. $P\left(r, \lambda, e_{1}\right)$ is higher than $P\left(r, \lambda, e_{2}\right)$.

In the state $e_{2}$, the present value of cash flows is at par, so $\xi\left(\overline{r_{0}}, \overline{\lambda_{0}}, \overline{X_{0}}\right)=1$. The prepayment option price is $P\left(\overline{r_{0}}, \overline{\lambda_{0}}, \overline{X_{0}}\right)=0.1033$. Therefore the loan value equals $\xi\left(\overline{r_{0}}, \overline{\lambda_{0}}, \overline{X_{0}}\right)-P\left(\overline{r_{0}}, \overline{\lambda_{0}}, \overline{X_{0}}\right)=0.8967$. If the bank decides to include the prepayment option in the initial contractual margin $\overline{\overline{\rho_{0}}}$ (cf. Remark 2) the loan value will equal par for $\overline{\overline{\rho_{0}}}=1199 \mathrm{bps}$ in the state $e_{2}$, significantly higher than $\overline{\rho_{0}}$.

REMARK 7. In all examples the sensitivity with respect to $r$ is less critical than the sensitivity with respect to $\lambda$.

4.3. Application 3 : $N=2$ regimes with a non-zero correlation $\rho$. Consider a loan with the same parameters as in Section 4.2 and assume a non-zero correlation between the Brownian motions in the dynamics of $\lambda_{t}$ and $r_{t}$; we take $\rho=-0.5$.

The initial contractual margin is found to be $\overline{\rho_{0}}=854 \mathrm{bps}$ for $\overline{X_{0}}=e_{2}$. Even with 

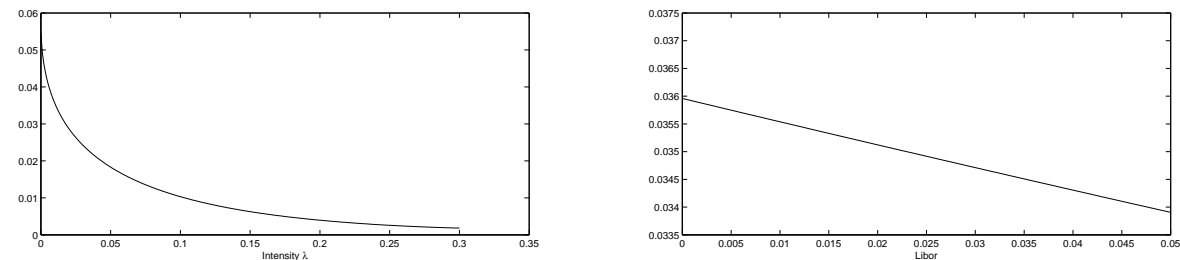

Fig. 4.5. Prepayment option value as a function of the intensity (left : $P\left(\overline{r_{0}}, \lambda\right)$ ) and LIBOR (right: $P\left(r, \overline{\lambda_{0}}\right)$ ) for the inputs in Section 4.1.
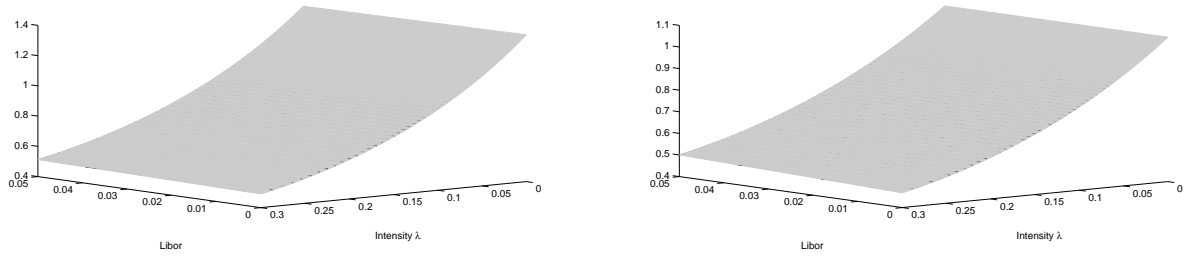

Fig. 4.6. $\xi(r, \lambda, X)$ as a function of the intensity and the LIBOR for the inputs in Section 4.2. Left: regime $X=e_{1}$; Right: regime $X=e_{2} . \xi\left(r, \lambda, e_{1}\right)$ is higher than $\xi\left(r, \lambda, e_{2}\right)$ according to the degradation of the credit quality ( $\lambda$ parameters) and the degradation of the access to money market (funding issues). It converges to 0 at infinity.

such a substantial correlation, there is only a $4 b p s$ increase with respect to the example in Section 4.2 (that had null correlation). The optimal boundaries are practically the same in both examples, as illustrated in Figure 4.9. The prepayment option price is $P\left(\overline{r_{0}}, \overline{\lambda_{0}}, \overline{X_{0}}\right)=0.1026$. Therefore the loan value equals $\xi\left(\overline{r_{0}}, \overline{\lambda_{0}}, \overline{X_{0}}\right)-P\left(\overline{r_{0}}, \overline{\lambda_{0}}, \overline{X_{0}}\right)=$ 0.8974 .

4.4. Application $4:$ impact of the end of a recession. In the Section 4.2 we considered that the loan originates in a state of recession but the bank uses a multi-regime model. We consider in this section a simpler case with a unique regime $(N=1)$ which is a recession regime. All parameters are the parameters of Section 4.2 for $X=e_{2}$.

The initial contractual margin is found to be $\overline{\rho_{0}}=1,204 b p s$ which is a sharp increase with respect to $851 \mathrm{bps}$ found in Section 4.2. On the other hand the prepayment option price is lower: $P\left(\overline{r_{0}}, \overline{\lambda_{0}}\right)=0.01855$ and the loan value equals $\xi\left(\overline{r_{0}}, \overline{\lambda_{0}}\right)-$ $P\left(\overline{r_{0}}, \overline{\lambda_{0}}\right)=0.98145$.

Numerical results illustrated in Figure 4.10 indicate that the domain is divided in a continuation region and an exercise region. Thus, in this situation, a model with one unique regime indicates that the client can prepay during a recession.

4.5. Discussion and interpretation of the numerical results. Several conclusions can be drawn from the examples above. First of all, the prepayment option has a non-negligible impact on the loan value and as such it should be taken into account and its risk assessed.

Secondly, the presence of a multi-regime dynamics can change dramatically the exercise and continuation regions: while a single-regime recession (Section 4.4) will display a exercise region, a two-regime model (Section 4.2) displays an exercise region only in the "normal" regime and none during recession time. This is completely consistent with actual banking practice : clients seldom prepay during recessions. 


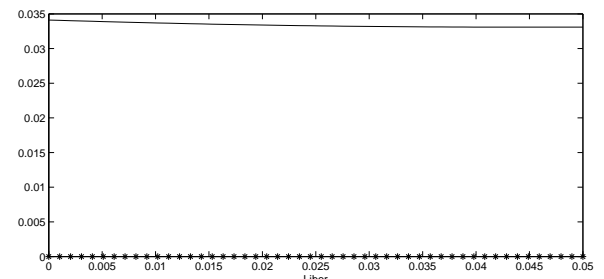

Fig. 4.7. The optimal boundary: $\Gamma_{1}^{*}$ in regime $X=e_{1}$ (solid line) and $\Gamma_{2}^{*}$ in regime $X=e_{2}$ (asterisk line) as function of the LIBOR $r$ for the inputs in Section 4.2. Recall that the continuation region is above the boundary and the exercise region below the boundary. For regime $e_{1}$ two regions appear : the continuation region and the exercise region while for regime $e_{2}$ only the continuation region is present. The borrower will only prepay in regime $e_{1}$ after the crisis is over.
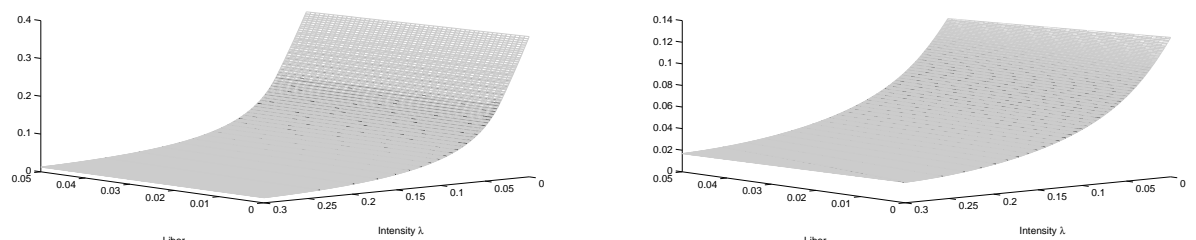

FIG. 4.8. The price of the prepayment option $P(r, \lambda, X)$ as function of the intensity and LIBOR for the inputs in Section 4.2. Left: regime $X=e_{1}$; Right: regime $X=e_{2}$.

Thus the conclusions of the single-regime model are misleading regarding the (optimal) behavior of the clients.

On the contrary, it is probable that some clients will exercise their prepayment option when the economy recovers. The model proposes a quantitative framework to explain when this may happen as a function of the credit spread $\lambda_{t}$ of the client and of the short rate $r_{t}$.

Finally, the value $r_{t}$ and the correlation $\rho$ between the CIR dynamics of $r_{t}$ and $\lambda_{t}$ play a secondary role in the qualitative properties of the prepayment option.

Appendix A. Details of the computations in equation (3.7).

We integrate by parts:

$$
\begin{aligned}
& \iint_{\left(\mathbb{R}_{+}\right)^{2}} \partial_{r \lambda} f g \sqrt{r \lambda} h d r d \lambda=\left.\int_{\mathbb{R}_{+}} \partial_{\lambda} f g \sqrt{r \lambda} h\right|_{r=0} ^{r=\infty} d \lambda-\iint_{\left(\mathbb{R}_{+}\right)^{2}} \partial_{\lambda} f \partial_{r}(g \sqrt{r \lambda} h) d r d \lambda \\
& =0-\iint_{\left(\mathbb{R}_{+}\right)^{2}} \partial_{\lambda} f \partial_{r} g \sqrt{r \lambda} h d r d \lambda+\partial_{\lambda} f g \partial_{r}(\sqrt{r \lambda} h) d r d \lambda \\
& =-\iint_{\left(\mathbb{R}_{+}\right)^{2}} \partial_{\lambda} f \partial_{r} g \sqrt{r \lambda} h d r d \lambda-\left.\int_{\mathbb{R}_{+}} f g \partial_{r}(\sqrt{r \lambda} h)\right|_{\lambda=0} ^{\lambda=\infty} d r \\
& +\iint_{\left(\mathbb{R}_{+}\right)^{2}} f \partial_{\lambda}\left(g \partial_{r}(\sqrt{r \lambda} h)\right) d r d \lambda=-\iint_{\left(\mathbb{R}_{+}\right)^{2}} \partial_{\lambda} f \partial_{r} g \sqrt{r \lambda} h d r d \lambda \\
& \left.+\iint_{\left(\mathbb{R}_{+}\right)^{2}} f g \partial_{\lambda r}(\sqrt{r \lambda} h) d r d \lambda+\iint_{\left(\mathbb{R}_{+}\right)^{2}} f \partial_{\lambda} g \partial_{r}(\sqrt{r \lambda} h)\right) d r d \lambda
\end{aligned}
$$




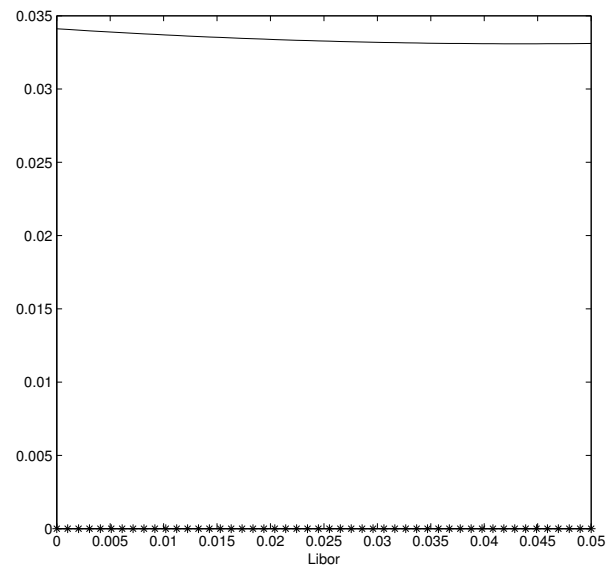

Fig. 4.9. The optimal boundary: $\Gamma_{1}^{*}$ in regime $X=e_{1}$ (solid line) and $\Gamma_{2}^{*}$ in regime $X=e_{2}$ (asterisk line) as function of the LIBOR $r$ for the inputs in Section 4.3. Recall that the continuation region is above the boundary and the exercise region below the boundary. The results are similar to the situation with no correlation.

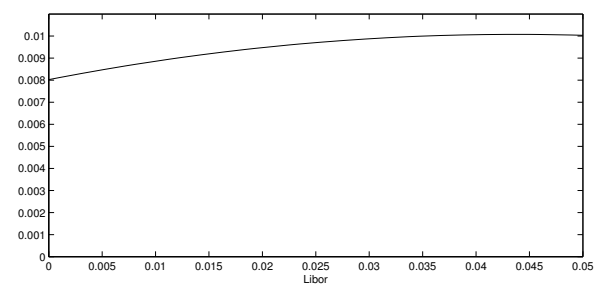

FIG. 4.10. The optimal boundary: $\Gamma^{*}$ as function of the LIBOR $r$ for the inputs in Section 4.4 . Recall that the continuation region is above the boundary and the exercise region below the boundary. In particular the exercise region is not empty.

The first two terms are already in convenient form. For the last one we write:

$$
\begin{aligned}
& \left.\iint_{\left(\mathbb{R}_{+}\right)^{2}} f \partial_{\lambda} g \partial_{r}(\sqrt{r \lambda} h)\right) d r d \lambda=\left.\int_{\mathbb{R}_{+}} f g \partial_{r}(\sqrt{r \lambda} h)\right|_{\lambda=0} ^{\lambda=\infty} d r \\
& \left.\left.-\iint_{\left(\mathbb{R}_{+}\right)^{2}} g \partial_{\lambda}\left(f \partial_{r}(\sqrt{r \lambda} h)\right)\right) d r d \lambda=0-\iint_{\left(\mathbb{R}_{+}\right)^{2}} g \partial_{\lambda} f \partial_{r}(\sqrt{r \lambda} h)\right) d r d \lambda \\
& -\iint_{\left(\mathbb{R}_{+}\right)^{2}} g f \partial_{\lambda r}(\sqrt{r \lambda} h) d r d \lambda .
\end{aligned}
$$

One adds now the term $\left.\iint_{\left(\mathbb{R}_{+}\right)^{2}} f \partial_{\lambda} g \partial_{r}(\sqrt{r \lambda} h)\right) d r d \lambda$ to each member of this identity to write:

$$
\begin{aligned}
& \left.\iint_{\left(\mathbb{R}_{+}\right)^{2}} f \partial_{\lambda} g \partial_{r}(\sqrt{r \lambda} h)\right) d r d \lambda \\
& \left.=\iint_{\left(\mathbb{R}_{+}\right)^{2}} \frac{f \partial_{\lambda} g-g \partial_{\lambda} f}{2} \partial_{r}(\sqrt{r \lambda} h)\right) d r d \lambda-\iint_{\left(\mathbb{R}_{+}\right)^{2}} \frac{f g \partial_{\lambda r}(\sqrt{r \lambda} h)}{2} d r d \lambda .
\end{aligned}
$$


We obtain thus (3.7).

Appendix B. Regularity properties for $\xi, \chi, P_{\Omega^{*}}$.

B.1. Regularity for $\xi$. Recall first equation (2.18) that gives an uniform (in $r, \lambda, X) L^{\infty}$ bound for $\xi$. Also note that equation (2.24) is pointwise satisfied for all $r>0, \lambda>0, X \in E$.

In order to prove further regularity properties for $\xi$ two distinct ways are possible: the probabilistic interpretation or the PDE. We will prefer the PDE version in order to be more close to the results required in Section 3.

Let us first fix $X=e_{k}$ and some $r>0, \lambda>0$. Then equation (2.24) is true in some open ball $B$ around $r>0, \lambda>0$ of radius $\min \{r, \lambda\} / 2$. It can be written, with convention $\xi_{k}(r, \lambda)=\xi_{k}\left(r, \lambda, e_{k}\right)$, as:

$$
\begin{aligned}
& -\mathcal{A}_{k} \xi_{k}+\left(r+\lambda+l_{k}\right) \xi_{k}=F_{k}, \forall r, \lambda \in B \\
& \left.\xi_{k}(r, \lambda)\right|_{\partial B}=G_{k} .
\end{aligned}
$$

where $F_{k}, G_{k}$ are functions (depending on $\xi$ ) bounded in $L^{\infty}$ by a given, known, constant $M$.

From the definition of the ball $B$ the operator $\mathcal{A}_{k}$ is strictly coercive on $B$. Thus $\xi_{k}$ is solution of a strictly elliptic problem. Standard PDE results imply that $\xi_{k}(r, \lambda) \in$ $W^{2, \infty}(B)$ i.e., the space of functions that have two $L^{\infty}$ derivatives. But then, as $F_{k}$ and $G_{k}$ are defined in terms of $\xi_{k}$ they are also in $W^{2, \infty}$. The process is then bootstrapped to obtain, together with standard Sobolev embeddings that $\xi_{k}$ is $C^{\infty}$ at $(r, \lambda)$. An alternative proof is to use the tangent process (see [28] Theorem 39 chapter $\mathrm{V})$ to obtains bounds for the derivatives with respect to $r$ and $\lambda$.

Let us now compute, since $\xi$ is regular locally, $a_{\star}(\xi, \xi)$ according to its definition in equation (3.3)

$$
\begin{aligned}
& \langle\xi, \xi\rangle_{\star}=a_{\star}(\xi, \xi)-b_{\star}(\xi, \xi)=a_{\star}(\xi, \xi)-0 \\
& =\sum_{k=1}^{N} \iint_{\left(\mathbb{R}_{+}\right)^{2}}\left(-\mathcal{A}^{\mathcal{R}}(\xi)\right)\left(r, \lambda, e_{k}\right) \xi\left(r, \lambda, e_{k}\right) \mu_{k} d r d \lambda \\
& =\sum_{k=1}^{N} \iint_{\left(\mathbb{R}_{+}\right)^{2}}\left(r+\overline{\rho_{0}}\right) K \xi\left(r, \lambda, e_{k}\right) \mu_{k} d r d \lambda<C \sum_{k=1}^{N} \iint_{\left(\mathbb{R}_{+}\right)^{2}}(r+1) \mu_{k} d r d \lambda,
\end{aligned}
$$

for some constant $C$. If suffices now to recall that the first order moment of $\mu_{k}$ is finite i.e., $\sum_{k=1}^{N} \iint_{\left(\mathbb{R}_{+}\right)^{2}} r \mu_{k} d r d \lambda<\infty$ (see appendix C); we conclude that $\xi \in \mathcal{H}_{\star}$.

B.2. Regularity for $\chi$. Note that $\chi=\mathbf{1}_{\xi>K}$. Moreover the derivatives of $\xi$ and $\chi$ coincide on the set $\{\xi>K\}$ and elsewhere the derivatives are zero. Finally, on $\{\xi>K\}, \xi>\chi \geq 0$. Thus $\langle\chi, \chi\rangle_{\star} \leq\langle\xi, \xi\rangle_{\star}<\infty$ thus $\chi \in \mathcal{H}_{\star}$.

B.3. Regularity for $P_{\Omega^{*}}$. From (2.34) one obtains that

$$
P(r, \lambda, X) \leq K\left(1+\overline{\rho_{0}}\right), \forall r, \lambda, X .
$$

Note that $P_{\Omega^{*}}=P_{\Omega^{*}} \mathbf{1}_{P_{\Omega^{*}}>\chi}+P_{\Omega^{*}} \mathbf{1}_{P_{\Omega^{*}}=\chi}$ and recall that on $\left\{P_{\Omega^{*}}>\chi\right\}$ we have $\mathcal{A}^{\mathcal{R}} P_{\Omega^{*}}=0$; thus

$$
\begin{aligned}
& a_{\star}\left(P_{\Omega^{*}}, P_{\Omega^{*}}\right)=a_{\star}\left(P_{\Omega^{*}} \mathbf{1}_{P_{\Omega^{*}}>\chi}+P_{\Omega^{*}} \mathbf{1}_{P_{\Omega^{*}}=\chi}, P_{\Omega^{*}}\right)=0+a_{\star}\left(P_{\Omega^{*}} \mathbf{1}_{P_{\Omega^{*}}=\chi}, P_{\Omega^{*}}\right) \\
& =a_{\star}\left(\chi \mathbf{1}_{P_{\Omega^{*}}=\chi}, \chi \mathbf{1}_{P_{\Omega^{*}}=\chi}\right)=\left\langle\chi \mathbf{1}_{P_{\Omega^{*}}=\chi}, \chi \mathbf{1}_{P_{\Omega^{*}}=\chi}\right\rangle_{\star} \leq\langle\chi, \chi\rangle_{\star}<\infty .
\end{aligned}
$$


Hence $P_{\Omega^{*}} \in \mathcal{H}_{\star}$.

\section{Appendix C. Some properties of $\mu$.}

Similar techniques as in previous sections allow to prove that $\mu_{k}$ is $C^{\infty}$. We will only prove that the first order moment with respect to $r$ is finite, all others follow the same lines of proof. Recall that since $\mu$ is a stationary distribution, by ergodicity :

$$
\sum_{k=1}^{N} \iint_{\left(\mathbb{R}_{+}\right)^{2}} r \mu_{k} d r d \lambda=\lim _{T \rightarrow \infty} \frac{\int_{0}^{T} \mathbb{E}\left(r_{t}\right) d t}{T} .
$$

The equation is true irrespective of the starting point $r_{0}, \lambda_{0}, X_{0}$. Denote $m_{t}=\mathbb{E}\left(r_{t}\right)$. An application of the Itto formula gives that

$$
\frac{d}{d t} m_{t}=\mathbb{E} \gamma_{r}\left(X_{t}\right)\left(\theta_{r}\left(X_{t}\right)-r_{t}\right), m_{0}=r_{0} .
$$

Of course $m_{t} \geq 0, \forall t$. The process $X_{t}$ is piecewise constant. In particular $\theta_{r}\left(X_{t}\right)$ takes a finite number of values, let us denote $M^{-}=\min _{k} \theta_{r}\left(e_{k}\right) \gamma_{r}\left(e_{k}\right), M^{+}=$ $\max _{k} \theta_{r}\left(e_{k}\right) \gamma_{r}\left(e_{k}\right), \gamma_{r, \max }=\max _{k} \gamma_{r}\left(e_{k}\right), \gamma_{r, \min }=\min _{k} \gamma_{r}\left(e_{k}\right)$. Then for all $t$ :

$$
\forall t \geq 0: \frac{d}{d t} m_{t} \in\left[\gamma_{r, \max }\left(\frac{M^{-}}{\gamma_{r, \max }}-m_{t}\right), \gamma_{r, \min }\left(\frac{M^{+}}{\gamma_{r, \min }}-m_{t}\right)\right], m_{0}=r_{0} .
$$

Then the distance from $m_{t}$ to the interval $\left[\frac{M^{-}}{\gamma_{r, \text { max }}}, \frac{M^{+}}{\gamma_{r, \text { min }}}\right]$ is decreasing hence $m_{t}$ is

bounded by some constant $C$. Therefore $\lim _{T \rightarrow \infty} \frac{\int_{0}^{T} \mathbb{E}\left(r_{t}\right) d t}{T} \leq C<\infty$ which gives the conclusion.

\section{REFERENCES}

[1] Aurélien Alfonsi, On the discretization schemes for the CIR (and Bessel squared) processes, Monte Carlo Methods and Applications, 11 (2005), pp. 355-384.

[2] Alain Bensoussan, On the theory of option pricing, Acta Applicandae Mathematicae, 2 (1984), pp. 139-158. 10.1007/BF00046576.

[3] Tomasz R. Bielecki and Marek Rutkowski, Credit Risk: Modeling, Valuation and Hedging, Springer-Verlag Berlin and Heidelberg GmbH and Co. K, 2010.

[4] J. Frédéric Bonnans, J. Charles Gilbert, Claude Lemaréchal, and Claudia A. Sagastizábal, Numerical optimization, Universitext, Springer-Verlag, Berlin, second ed., 2006. Theoretical and practical aspects.

[5] John Buffington and Robert J. Elliott, American options with regime switching, International Journal of Theoretical and Applied Finance, 5 (2009), pp. 497-514.

[6] Cox John C., Ingersoll Jonathan E., and Ross Stephen A., A theory of the term structure of interest rates, Econometrica, 53 (1985), p. 385.

[7] Alex Cerny, Mathematical Techniques in Finance: Tools for Incomplete Markets (Second Edition), Princeton University Press, Princeton University Press, 2009.

[8] Yong Chen, Michael Connolly, Wenjin Tang, and Tie Su, The value of mortgage prepayment and default options, Journal of Futures Markets, 29 (2009), pp. 840-861.

[9] Marc Chesney, Robert J. Elliott, and Rajna Gibson, Analytical solutions for the pricing of American bond and yield options, Mathematical Finance, 3 (1993), pp. 277-294.

[10] Didier Cossin and Lu Hongze Abraham, Pricing prepayment option in $C$ \& $I$ loans at origination. University of Lausanne - School of Economics and Business Administration (HEC-Lausanne).

[11] John Detemple, American-Style Derivatives: Valuation and Computation, Chapman \& Hall / CRC Financial Mathematics Series, Chapman \& Hall, 2006.

[12] Georges Dionne, Genevieve Gauthier, Khemais Hammami, Mathieu Maurice, and Jean-Guy Simonato, A reduced form model of default spreads with Markov-switching macroeconomic factors, Journal of Banking and Finance, 35 (2011), pp. $1984-2000$. 
[13] John W. Eaton, David Bateman, and Soren Hauberg, Gnu Octave Version 3. 0. 1 Manual: A High-Level Interactive Language for Numerical Computations, CreateSpace, 2009.

[14] Robert J. Elliott and Tak Kuen Siu, On Markov-modulated exponential-affine bond price formulae, Applied Mathematical Finance, 16 (2009), pp. 1-15.

[15] XIN Guo, An explicit solution to an optimal stopping problem with regime switching, J. Appl. Probab., 38 (2001), pp. 464-481.

[16] Frederic Hecht, Olivier Pironneau, Jacques Morice, Antoine le Hyaric, and Kohji Oнtsuka, FreeFem++. http://www.freefem.org/ff++/, 2012.

[17] Jimmy E. Hillard, James B. Kau, and Carlos V. Slawson, Valuing prepayment and default in a fixed-rate mortgage: A bivariate binomial options pricing technique, Real Estate Economic, 26 (1998), pp. 431-468.

[18] Yunqing Huang, Peter A. Forsyth, and George Labahn, Methods for pricing American options under regime switching, SIAM J. Sci. Comput., 33 (2011), pp. 2144-2168.

[19] Patrick Jaillet, Damien Lamberton, and Bernard Lapeyre, Variational inequalities and the pricing of American options, Acta Applicandae Mathematicae, 21 (1990), pp. 263-289. 10.1007/BF00047211.

[20] Adriann Jobert and Leonard C. G. Rogers, Option pricing with Markov-modulated dynamics, SIAM J. Control Optim, 44 (2006), pp. 2063-2078.

[21] David Kinderlehrer and Guido Stampacchia, An Introduction to Variational Inequalities and Their Applications, no. vol. 88 in Pure and Applied Mathematics, Academic Press, 1980.

[22] Damien Lamberton and Bernard Lapeyre, Introduction to stochastic calculus applied to finance, 2nd edition, Chapman \& Hall/ CRC, 2008.

[23] David Lando, Credit Risk Modeling, Princeton University Press, 2004.

[24] Xue Liang and Guojing Wang, On a reduced form credit risk model with common shock and regime switching, Insurance: Mathematics and Economics, 51 (2012), pp. 567 - 575.

[25] Rogemar S. Mamon and Marianito R. Rodrigo, Explicit solutions to European options in a regime-switching economy, Operations Research Letters, 33 (2005), pp. 581-586.

[26] Bernt Oksendal, Stochastic differential equations, Universitext, Springer, Berlin ; Heidelberg [u.a.], 6. ed. ed., 2007.

[27] Timothee Papin and Gabriel Turinici, Valuation of the Prepayment Option of a Perpetual Corporate Loan, Abstract and Applied Analysis, 2013 (2013), p. 960789.

[28] Philip E. Protter, Stochastic Integration and Differential Equations: Version 2.1, Applications of Mathematics Series, Springer, 2005.

[29] Eduardo S. Schwartz and Walter N. Torous, Mortgage prepayment and default decisions: A Poisson regression approach, American Real Estate and Urban Economics Association, 21 (1993), pp. 431-449.

[30] TAK KUEN SiU, Bond pricing under a markovian regime-switching jump-augmented vasicek model via stochastic flows, Applied Mathematics and Computation, 216 (2010), pp. 3184 $-3190$.

[31] Tak Kuen Siu, Hailiang Yang, and John W. Lau, Pricing currency options under twofactor Markov-modulated stochastic volatility models, Insurance: Mathematics and Economics, 43 (2008), pp. $295-302$.

[32] Yongqing Xu And Yandong Wu, Perpetual American maximum options with Markov-modulated dynamics, Lithuanian Mathematical Journal, 51 (2011), pp. 106-119. 10.1007/s10986011-9111-y.

[33] David D. Yao, Qing Zhang, And Xun Yu Zhou, A regime-switching model for European options, in Stochastic Processes, Optimization, and Control Theory: Applications in Financial Engineering, Queueing Networks, and Manufacturing Systems, Houmin Yan, George Yin, and Qing Zhang, eds., vol. 94 of International Series in Operations Research \& Management Science, Springer US, 2006, pp. 281-300.

[34] Qing Zhang and Xin Guo, Closed-form solutions for perpetual American put options with regime switching, SIAM J. Appl. Math., 64 (2004), pp. 2034-2049.

[35] Nanxin Zhou and Rogemar Mamon, An accessible implementation of interest rate models with Markov-switching, Expert Systems with Applications, 39 (2012), pp. 4679 - 4689. 